\title{
Beschreibung des Echappements mit vollkommen freiem Pendel.
}

\author{
Von Ingenieur S. Ricfler.
}

In dem "Bericht über den Gang einer Riefler'schen Pendeluhr* (A. N. 3182) hat Herr Dr. Anding eine kurze Beschreibung meines Echappements mitgetheilt. Da jedoch keine Zeichnungen beigefugt sind, ohne welche das Verständniss wohl etwas schwer ist, so erlaube ich mir das Echappement an der Hand von Abbildungen nochmals etwas ausführlicher $z u$ beschreiben.

Die Gleichförmigkeit des Ganges einer Pendeluhr hängt hauptsächlich von zwei Factoren $a b$, erstens von der Vollkommenheit des Echappements, zweitens von der Wärmecompensation des Pendels.

Unter Echappement versteht man denjenigen Mechanismus der Uhr, welcher die treibende Kraft des Räderwerkes auf das Pendel überträgt, um diesem diejenige Energie wieder zuzuführen, welche dasselbe durch die bei seinen Schwingungen auftretenden Widerstände verliert.

Ein vollkommenes Echappement soll so beschaffen sein, dass Aenderungen in der Triebkraft des Räderwerkes, welche wegen der nicht $2 u$ vermeidenden Ungleichheiten der Rädereingriffe und der Zapfenreibung stets vorkommen, ohne nennenswerthen Einfluss auf die Gleichförmigkeit der Pendelschwingungen sind. Eine theoretische Ueberlegung, auf welche hier nicht năher eingegangen werden soll, ergab nir, dass dies dann der Fall ist, wenn das Echappement folgenden Constructionsbedingungen entspricht :

I) Das Pendel soll möglichst frei und unabhängig vom Räderwerk schwingen.

2) Der Antrieb des Pendels soll möglichst nahe an der Schwingungsaxe stattfinden.

3) Derselbe soll ausserdem nahe in dem Moment erfolgen, in welchem das Pendel die grösste lebendige Kraft besitzt, d. i. in der Mittellage.

4) Der Antrieb soll rasch, jedoch stossfrei, d. i. ohne Vibrationen des Pendels zu erzeugen, vor sich gehen.

Das bisher für astronomische Uhren am meisten angewendete Graham-Echappement erfullt die erste der genannten drei Bedingungen unter allen Echappements am unvollkommensten. Das Pendel steht hier nahezu während seiner ganzen Schwingung mit dem Räderwerk in Verbindung und wird durch die Ungleichheiten des Druckes und der Reibung beeinflusst. So hat u. A. Lamont durch Versuche festgestellt, dass ein Pendel, welches im freien Zustand genau Secunden schwingt, um 3 bis 4 Secunden täglich zurückbleibt, wenn es mit einem Uhrwerk mit GrahamEchappement in Verbindung gebracht wird, dass diese Retardation jedoch mit zunehmender Verdickung des Oels auf den Paletten bis auf das drei- und vierfache anwachsen kano.

Mehrfach, theils von namhaften Constructeuren (Winnerl, Denison, Nieberg etc.), ist daher versucht worden das Problem des freischwingenden Pendels zu lösen; allein keine dieser Constructionen hat eine ausgedehntere Anwendung bei astronomischen Uhren gefunden. Der erzielte Erfolg war ungenügend, hauptsächlich weil einerseits die Freiheit des Pendels stets nur unvollkommen erreicht wurde, andererseits aber auch weil diese Echappements den für den Pendelantrieb maassgebenden Bedingungen 2) und 3) nicht ausreichend genugen.

Inwieweit es mir nun gelungen ist das angestrebte Ziel zu erreichen, dürte aus der hier folgenden Beschreibung meines Echappements hervorgehen.

Bei diesem Echappement schwingt das Pendel vollkommen frei, da es mit dem Uhrwerk nur durch die Aufhängefeder in Verbindung steht. Der Antrieb erfolgt dadurch, dass die Aufhängefeder bei jeder Pendelschwingung durch das Räderwerk eine kleine Biegung erfährt und hierdurch ein wenig gespannt wird, und diese Spannkraft der Pendelfeder ist es, welche dem Pendel den Antrieb ertheilt. Da diese Biegung um eine Axe erfolgt, welche mit der Schwingungsaxe des Pendels zusammenfallt, und ausserdem jedesmal nahezu in dem Moment eintritt, in welchem das Pendel durch die Mittellage hindurchschwingt, so ist ausser der vollkommenen Freiheit des Pendels auch noch der grosse Vortheil erreicht, dass Ungleichheiten in der Kraftzufuhr vom Räderwerk keinen nachtheiligen Einfluss auf die Gleich. förmigkeit des Uhrganges haben. Ueberdies ist der Pendelantrieb auch noch von nahezu constanter Grösse, da die Pendelfeder, deren Biegungsspannkraft den Antrieb bewirkt, bei jeder Pendelschwingung un den gleichen Winkel gebogen wird, dessen Grösse durch die Begrenzung der Ankerbewegung gegeben und unabhängig von der Zugkraft ist.

In den Zeichnungen stellt Fig. I die Vorder-, Fig. 2 die Seitenansicht des Echappements im Maassstabe 5:6 und Fig. 3 dessen Ansicht von oben in natürlicher Grösse dar, wie dasselbe fur astronomische Uhren ausgefuhrt wird.

Fig. 4 und 5 sind Abbildungen der Pendelaufhängung in wirklicher Grösse mit Axe und Pendelfeder. 
$T T$ (Fig. I u. 2) ist ein an der rückseitigen Werkplatine $W$ der Uhr durch vier Schrauben $u \boldsymbol{u}$ festgeschraubter krăftiger Träger aus Metallguss, in welchem die beiden Lagersteine $P P$ befestigt sind, deren ebene Oberflächen, zwischen denen die Pendelaufhängung hindurchgeht, zusammen in einer horizontalen Ebene liegen.

Auf dieser Ebene liegt die Drehungsaxe a $a$ (Fig. 2) des Ankers $A$, welche durch die Messerschneiden der Stahlprismen $c c$ gebildet ist. Die für den ordnungsgemässen Eingriff des Ankers in die Gangräder $H$ und $R$ erforderliche Richtung erhält die Drehungsaxe des Ankers durch die Körnerspitzen der Schrauben $K K^{1}$, welche jedoch, weon das Pendel $B$ eingehängt ist, ein wenig zurückgeschraubt werden, damit sie das freie Spiel des Ankers nicht beeinträchtigen.

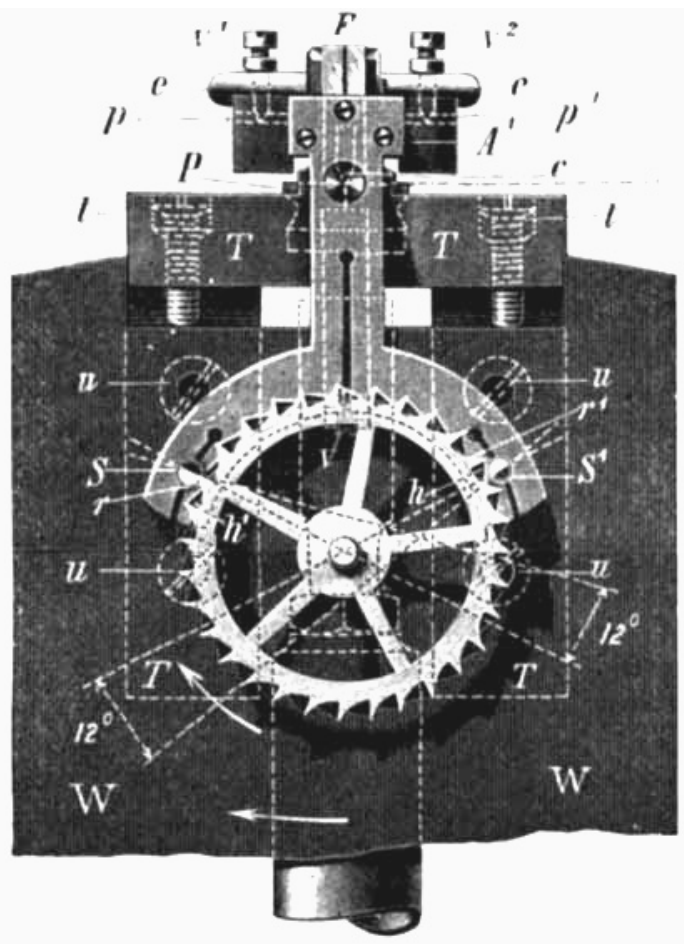

Fig. 1. M - $5: 6$.

$F F^{1}$ ist die auf das Ankerstück $A^{1} A^{1}$ aufgesetzte Pendelaufhängung mit der Pendelfeder $i i$, deren Biegungsaxe genau mit der Drehungsaxe a $a$ des Ankers zusammenfällt.

Das Gangrad ist ein Doppelrad und besteht aus dem Hebungsrad $H$ (Fig. I u. 2) und dem etwas grösseren Ruherad $R$. Die Zähne $h h^{1}$ des ersteren bewirken mit ihren schrägen Flächen die Hebung, die Zähne $r r^{1}$ des letzteren bilden mit ihren radialen $F$ lächen die Ruhen.

$S$ und $S^{1}$ sind die Hebe- und zugleich Ruhepaletten des Ankers. Dieselben sind cylindrisch, jednch am vorderen Ende bis zur Cylinderaxe abgeflacht.

An der Cylinderfläche findet die Hebung des Ankers durch die Zähne des Hebungsrades $H$ statt, an den ebenen Flächen erfolgt die Ruhe durch die Zähne des Ruherades $R$.

Das Spiel des Echappements ist nun folgendes: Fig. I stellt dasselbe in dem Momente dar, in welchem das Pendel sich in der Ruhelage befindet und der Zahn $r$ des Ruherades auf der ebenen Fläche der Palette $S$ aufruht.

Schwingt nun das Pendel in der Richtung des Pfeils nach links aus, so bleibt die Pendelfeder $i$ zunächst noch gerade gestreckt und die Schwingung findet anfänglich um die Schneidenaxe $a$ a des Ankers statt. Der Anker $A$ wird, weil er durch die Pendelfeder $i i$ mit dem Pendel in Verbindung steht, diese Schwingung des Pendels soweit nitmachen, bis die Zahnspitze des Ruheradzahns r (Fig. I) von der Ruhefläche der Palette $S$ herabfällt. - Das Pendel hat bis dahin einen Bogen (Hebungsbogen) von etwa $1 / 4^{\circ}$ zurück. gelegt. - In diesem Moment ist die Cylinderflache der Palette $S^{1}$ an den Hebezahn $h$ des Hebungsrades bis auf den erforderlichen Spielraum herangerückt, die Räder drehen sich in der Pfeilrichtung, bis der Ruhezahn $r^{1}$ allf der

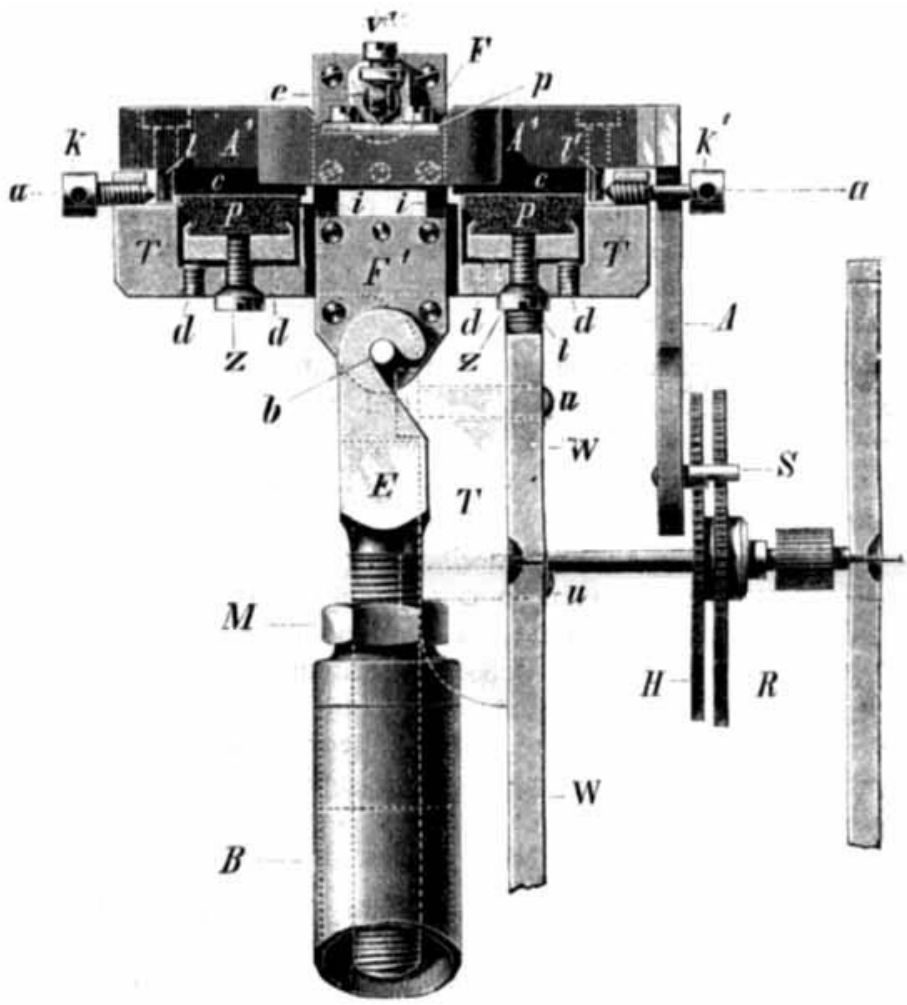

Fig. 2. $M=5: 6$.

ebenen Fläche der Palette $S^{1}$ aufliegt, und der Hebungszahn $h$ bewirkt während dieser Drehung die Hebung, d. $h$. derselbe drängt die Palette $S^{1}$ zurück und bewegt dadurch den Anker in der der Pendelschwingung entgegengesetzten Richtung.

Durch diese vom Räderwerk bewirkte Drehbewegung des Ankers hat die Pendelfeder $i i$ eine kleine Biegung um die Schwingungsaxe $a a$ und damit eine geringe Spannung erfahren, welche dem Pendel den Antrieb ertheilt.

Das Pendel folgt jedoch nicht sofort der antreibenden Kraft, sondern vollendet zunächst noch seine Schwingung nach links, nunmehr um die Biegungsaxe der Pendelfeder schwingend, wobei der Anker in Ruhe bleibt. Der betreffende Ergänzungsbogen beträgt bei astronomischen Uhren $1-114^{\circ}$. 
Bei der Rückkehr des Pendels wird, nachdem dasselbe die Mittellage nach rechts überschritten hat, der in. zwischen auf $S^{1}$ aufgeruhte Zahn $r^{\prime}$ frei und eine neue Hebung findet auf der anderen Seite durch den Zahn l't statt.

In der Abbildung sind noch verschiedene kleine Constructionstheile sichtbar, welche bisher nicht erwähnt worden sind. Dieselben haben mit der eigentlichen Function des Fchappements nichts zu thun, sondern sind lediglich Regulirvorrichtungen fur die genaue und bequeme Montirung des Echappements.

Die konische Schraube ${ }^{\prime}$ (Fig. I) dient zur Einstellung der Weite des Ankers, während die Tiefe des Ankereingriffes in die Gangräder durch die Schrauben $t t$ eingestellt wird.

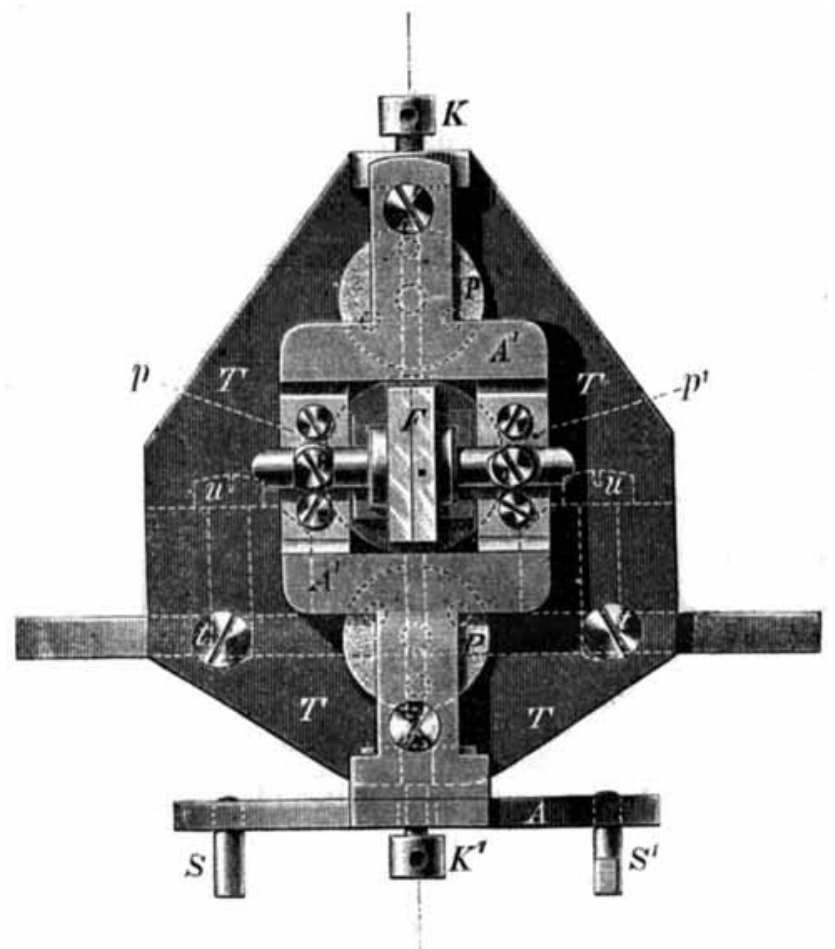

Fig. 3. M - , 1

Durch die Schrauben $\boldsymbol{v}^{1} \boldsymbol{v}^{2}$ der Pendelaufhängung, welche durch kleine Gegenmuttern festgestellt werden können, wird die Höhenlage der Pendelaufhängung eingestellt dergestalt, dass die Biegungsaxe det Pendelfeder $i$ i mit der Schneidenaxe als der Drehungsaxe $a$ a des Ankers zusammenfállt. Zugleich wird durch diese Schrauben auch der gleichmässige Abfall des Pendels regulirt.

Die Lagerschrauben $\gamma^{\prime 1} z^{2}$ der Pendelaufhängung ruhen mit ihren konischen Stirnflächen nicht direct auf dem Ankerstuck $A^{1} A^{1}$, sondern auf dunnen, mit entsprechenden Vertiefungen versehenen Lagerplättchen $p p^{1}$, welche auf das Ankerstück $A^{1} A^{1}$ aufgeschraubt sind, jedoch einigen Spielraum in den Schraubenlöchern haben. Dadurch kann die genaue Uebereinstimmung der Schneidenaxe a mit der Biegungsaxe der Pendelfeder in horizontaler Richtung bewirkt werden. $l$ und $l^{1}$ (Fig. 2) sind eingeschraubte Stahlstifte mit seitlichen Hohlkörnern, in welche die Körnerspitzen der Richtungsschrauben $K^{\prime} K^{\prime}$ eingreifen.

Die Lagersteine $P P$ ruhen mit ihren Messingfassungen auf je drei Druckschrauben $d$ auf, welche im Pendelträger $Y$ ihr Gewinde haben. Durch die Zugschraube $Z$ werden sie in der erforderlichen Lage festgehalten.

Wie leicht ersichtlich ist, bestehen die Widerstände, welche durch die Verbindung des Pendels mit dem Uhrwerk auf das Pendel einwirken, nur in der Axenreibung des Ankers und in dem Auslüsungswiderstand, welcher bei dem Herabgleiten der Zähne des Ruherades von den Rukeflächen der Paletten stattfindet. Beide Widerstände sind aber ausserst gering und tiberdies von sehr constanter Grösse.

Was zunächst die Axenreibung des Ankers betrifft, so besteht dieselbe nur aus der verschwindend kleinen wälzenden Reibung der Stahlschneiden $c c$ auf den vollkommen ebenen und sehr harten Lagersteinen $P P$. Sie wirkt über. dies nur einen kurzen Moment, in welchem das Pendel durch die Mittellage hindurchschwingt, also in dem nur $12^{\circ}$ betragenden Theil der Pendelschwingung, in welchem das. selbe die grösste Geschwindigkeit besitzt, auf das Pendel ein. In dem weitaus grössten Theil des Schwingungsbogens schwingt das Pendel um die Axe der Pendelfeder.

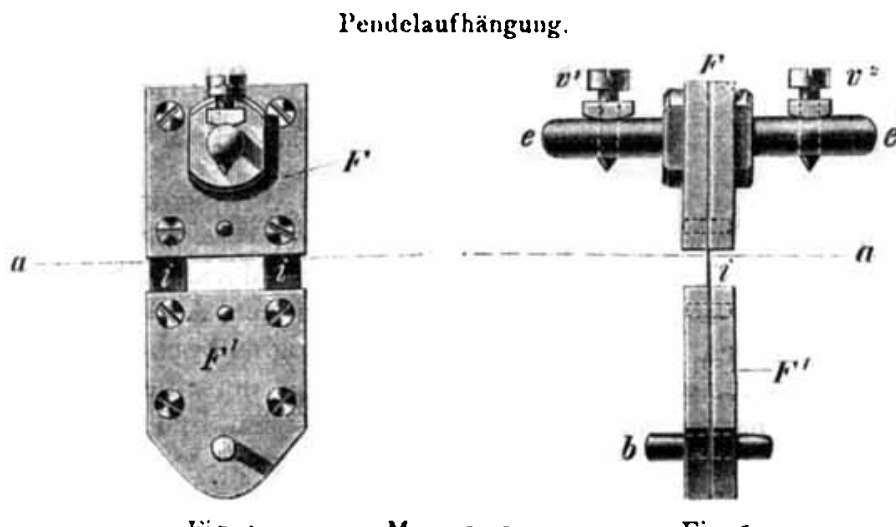

Fig. 4

$\mathbf{M}-\mathbf{I}: \mathbf{1}$.

Fig. 5

Auch der Auslösungswiderstand auf den Steinpaletten $S$ und $S^{\prime}$ ist sehr nahe gleich Null, weil die Ruheflächen nicht radial gestellt sind, sondern mit dem Radius der Gangräder einen Wiakel von etwa 10 bis $12^{\circ}$ bilden, welcher der Grösse des Reibungswinkels zwischen Stein und Messing gleichkommt.

Die Paletten sind also auf Schub, nicht wie beispielsweise beim Anker der Taschenuhr auf Zug, eingestellt: Die Gefahr einer unzeitigen Auslösung ist dabei ausgeschlossen, weil die Paletten durch die Spannung, welche die Pendelfeder bei dem Ausschwingen des Pendels erfährt, an die Zähne des Hebungsrades angedrückt werden.

Die Grösse des Schwingungsbogens des Pendels hängt bei diesem Echappement einerseits von der Steigung der Zähne des Hebungstades und andererseits von der Spannkraft der Pendelfeder, also hauptsächlich von der Dicke det letzteren ab. Je dicker die Pendelfeder ist, desto grösser wird der Ausschlag des Pendels. 
Bei astronomischen Uhren betragt die Dicke der Pendelfeder $0.1 \mathrm{~mm}$ und der ganze Schwingungsbogen ist $21 / 8^{\circ}$; wird bei diesen Uhren jedoch ein elektrischer Contact angebracht, so ist es vortheilhaft den Schwingungsbogen bis $\mathrm{zu} 2^{3} \%^{\circ}$ oder $3^{\circ} \mathrm{zu}$ erhöhen, was durch eine Pendelfeder von $0.125 \mathrm{~mm}$ Dicke erreicht wird.

Nebenbei mag hier erwahnt werden, dass man bei Pracisionsthurmuhren, fur welche das Echappement nunmehr gleichfalls mit tuberraschend günstigem Erfolg angewendet wird, einen Schwingungsbogen von $4^{\circ}$ mit einer $0.2 \mathrm{~mm}$ dickeo Pendelfeder erbält.

Man wird aus der Beschreibung entnehmen, inwiefern die oben aufgestellten Bedingungen bei diesem Echappement erftillt sind und worin seine Haupteigenthtimlichkeiten bestehen :

1) Das Pendel schwingt vollkommen frei und unbeeinflusst vom Uhrwerk.

2) Der Pendelantrieb findet in der Schwingungsaxe statt, so dass der Antriebhebel die geringste irgendwie mögliche Lánge hat. Dieselbe beträgt nur Bruchtheile eines Millimeters, da die Biegung der Pendelfeder sich nur über cine so geringe Lainge erstreckt. 3) Der Antrieb findet nahe in dem Moment statt, in bleibt.

München 1893 Nov. 16. welchem das Pendel durch die Mittellage hindurchschwingt, also die grösste lebendige Kraft besitzt.

4) Da die Hebung des Ankers sehr rasch vor sich geht, so erfolgt auch der Antrieb sehr schnell.

Derselbe findet aber auch vollständig stossfrei statt, weil er nicht vom starren Pendelstab, sondern von einem elastischen Zwischenglied, der Pendelfeder, aufgenommen wird.

Aus diesen Gründen haben Ungleichheiten in der Kraftzufuhr und in den Auslösungswiderständen keinen störenden Einfluss von nennenswerther Grösse auf die Gleichförmigkeit des Uhrganges.

Der Ergànzungsbogen des Echappements ist bei astronomischen Uhren drei bis vier Mal so gross als der Hebungsbogen. Das Pendel ist deshalb in hohem Grade unempfind: lich gegen störende Einflusse mechanischer Art.

Die Anzahl der wirkenden Theile des Echappements ist geringer als bei irgend einem der bekannten Echappements. Dasselbe functionirt daher mit der grössten Sicherheit. Bemerkenswerth ist noch, dass die Uhren mit diesem Echappement einen sehr kräftigen Pendelschlag haben, sowie dass der Schwingungsbogen stets von nahezu gleicher Grösse

\section{Berichtigungen zu Leipziger Sternpositionen in Band 69 bis 104 der Astronomischen Nachrichten.}

Infolge einer Anfrage von Herrn Professor Kam in Schiedam habe ich eine Anzahl von Fixsternbeobachtungen, welche Dr. R. Engelmann am Leipziger Meridiankreis angestellt hat und deren Resultate sich in den $\mathbf{A}$. N. publicirt finden, durch Zurlickgehen auf die Originalbeobachtungen gepruft, da sich bezliglich der Richtigkeit derselben Zweifel erhoben hatten, die sich auch in fast allen Fallen als berechtigt erwiesen haben. Nachstehend gebe ich das Verzeichniss der gefundenen Fehler.

$$
\text { A. } \pi .69 .100 \text {. }
$$

Die drei Sterne

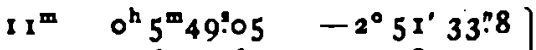

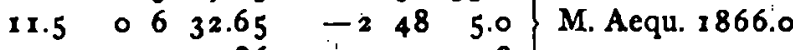

$$
\begin{aligned}
& \text { I1.5 } 0712.86 \\
& \begin{array}{lll}
-1 & 42 & 34.8
\end{array}
\end{aligned}
$$

existiren nicht am Himmel, Engelmann hat alle 3 Male dea Planeten (90) Antiope beobachtet. Die erhaltenen scheinbaren. Oerter des Planeten lauten

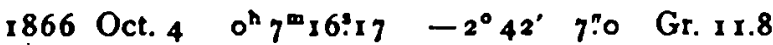

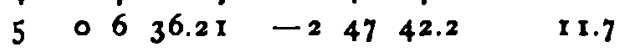

$$
\begin{aligned}
& 60552.60 \quad-25111.0 \quad \text { I1.0 }
\end{aligned}
$$

Die Positionen sind sehr unsicher, ganz besonders gilt dies von der von Oct. 5. Oct. 4 hatte Engelmann einzelne der sehr schlecht unter einander stimmenden Faden um ganze Secunden corrigirt; in Decl. war ein Reductionsfehler vorhanden; ausserdem ist hier ein Versehen von ' ${ }^{\prime}$ in der Kreisnotirung nicht ausgeschlossen. Oct. 6 hat Engelmann den beobachteten Mittelfaden um $-2^{3}$ corrigirt.

\section{A. R. 69.102 .}

\section{Statt}

$$
0^{h} 4^{m m} 32^{3}: 5^{2}+33^{\circ} 49^{\prime} \times 5^{\prime \prime 6} \text { (M. Aequ. 1866.0) }
$$

heisst die Position

$$
0^{\mathrm{h}} 4^{8^{\mathrm{m}}} 13^{3}: 85+33^{\circ} 49^{\prime} 15^{\mathrm{n} 6}
$$
gefuibrt.

Die Reduction auf den Mittelfaden war falsch aus-

Statt

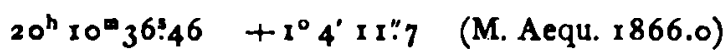

lautet die Position

$$
20^{\mathrm{h}} 10^{\mathrm{m}} 36: 46+14^{\mathrm{s}} 4^{\mathrm{m}} \cdot 2
$$

In Decl. lag ein Reductionsfehler vor. Dieser Stern befand sich am Ephemeridenorte von (4I) Daphne für Aug. 4 und war von Engelmann für diesen Planeten gehalten und beobachtet worden. Thatsächlich betrug indessen die Ephemeridencorrection ca. $+4: 8$ und $+4: 3$. Auch der Stern

$$
\text { 1866.0: } 20^{\mathrm{h}} 8^{\mathrm{m}} 27^{\mathrm{s}}: 64+0^{\circ} 3^{6} 5^{2}: 3
$$

war von Engelmann an Stelle von Daphne beobachtet worden, da er sich am 7. Aug. am Ephemeridenorte befand. Ein Fehler in der Position des letzteren Sterns, den Herr Professor Kam vermuthet, scheint mir ausgeschlossen $z u$ sein. Nach einer Notiz im Beobachtungsbuche geht diesem Stern ein Object $9: 7$ o:5 und 2:5 südlich voraus. Vop diesen beiden an Stelle von Daphne bestimmten Sternen finden sich schon A.N. 67 S. 344 vorläufige Positionen. 